\title{
Data Context
}

National Cancer Institute

\section{Source}

National Cancer Institute. Data Context. NCI Thesaurus. Code C89274.

Attributes from a data source that provide meaning that is relevant for annotation. 